\title{
CONSTRAINTS ON STELLAR EVOLUTION FROM OBSERVATIONS OF COMPACT OBJECT BINARIES
}

\author{
T. Bulik, ${ }^{1}$ R. Moderski, ${ }^{1}$ and K. Belczyński ${ }^{2}$
}

The masses of compact object (black hole, neutron star) binaries depend strongly on the parameters describing stellar evolution. Such masses or their functions can be measured using gravitational waves or through microlensing searches. We analyze an example of the varying common envelope efficiency and show the dependence of distributions of the measured chirp masses in gravitational waves mass ratios through microlensing taking into account the relevant selection effects.

We use the Star'Track binary population synthesis code (Belczyníski, Kalogera, Bulik, 2002) to calculate mass distributions of compact object binaries: double neutron stars (NSNS), black hole neutron star (BHNS), and double black hole binaries (BHBH). The mass distributions of compact object binaries depend on a number of parameters specified in the description of the StarTrack code. For the illustrative purpose we concentrate here on one parameter: the common envelope efficiency $\alpha_{C E} \lambda$ and discuss only the dependence of the results on this parameter.

Compact objec 1 binaries can be detected through gravitat ional wave's they (nit during inspiral. A primary observable is the chirp mass. A thorough analysis of the dependence of the observed chirp mass distribution in an Eucliclean space has already been published (Bulik \& Bol(críski. 2003). A detailed analysis (Bulik, Belczyński, Rudak 2003) shows that the cosmological effects and various star formation rate histories do not play a significant role in determining the shape of the distribution of observed chirp masses.

We also investigate the possibility of detecting compact object binaries through gravitational lensing. The masses of individual objects in a binary lens depend on the unknown position of the lens between the observer and the lensed star. However, the mass ratio can be estimated quite precisely. We use an analytical model of the Galactic potential to analyze the distribution of observed mass ratios in the microlensing events on stars in the galactic bulge (Bulik, Moderski, Belczyński 2003).

We acknowledge the KBN grants 5P03D01120 (TB), 5P03D00221 (RM), and the Lindheimer Fellowship (KB).

\section{REFERENCES}

Belczyński, K., Kalogera, V., \& Bulik, T. 2002, ApJ, 572, 407

Bulik, T. \& Belczyński, K. 2003, ApJ, 589, L37

Bulik, T., Belczyński, K. \& Rudak, B. 2003, A\&A, in press

Bulik, T., R. Moderski \& Belczyński, K. 2003, in preparation

\footnotetext{
${ }^{1}$ Nicolaus Copernicus Astronomical Center. Warsaw, Poland.

${ }^{2}$ Northwestern University, Evanston, IL, ISA.
} 